\title{
Survival-related risk score of lung adenocarcinoma identified by weight gene co-expression network analysis
}

\author{
HE WANG ${ }^{*}$, DI LU* ${ }^{*}$ XIGUANG LIU* ${ }^{*}$ JIANJUN JIANG, SIYANG FENG, XIAOYING DONG, \\ XIAOSHUN SHI, HUA WU, GANG XIONG, HAOFEI WANG and KAICAN CAI
}

Department of Thoracic Surgery, Nanfang Hospital, Southern Medical University, Guangzhou, Guangdong 510515, P.R. China

Received November 3, 2018; Accepted June 11, 2019

DOI: $10.3892 / \mathrm{ol} .2019 .10795$

\begin{abstract}
The present study aimed to identify the novel biomarkers and underlying molecular mechanisms of lung adenocarcinoma (LAC) to aid in its diagnosis, prognosis, prediction, disease monitoring and emerging therapies. Data from a total of 498 LAC samples were collected from The Cancer Genome Atlas and divided into two sets by stratified randomization based on pathological Tumor-Node-Metastasis stage. The training set was comprised of 348 samples and the validation set was comprised of 150 samples. A total of 123 samples from the training set for patients who completed follow-up were analyzed by weighted gene co-expression network analysis. A module was identified that contained 113 protein-coding genes that were positively associated with overall survival (OS). A least absolute shrinkage and selection operator (LASSO) Cox regression model was constructed and four survival-associated genes (OPN3, GALNT2, FAM83A and KYNU) were retained. Risk score, calculated by the linear combination of each gene expression multiplied by the LASSO coefficient, could successfully discriminate between patients with LAC exhibiting low and high OS time in both sets. The results from the present study indicate that this risk score may contribute to potential diagnostic and therapeutic strategies for LAC management.
\end{abstract}

\section{Introduction}

With an estimated 2.1 million new cases of lung cancer occurring in 2018 , accounting for $\sim 11.6 \%$ of all cancer diagnoses, lung cancer is the most frequently diagnosed type of cancer

Correspondence to: Dr Kaican Cai, Department of Thoracic Surgery, Nanfang Hospital, Southern Medical University, 1838 North Guangzhou Avenue, Guangzhou, Guangdong 510515, P.R. China E-mail: doc_cai@163.com

*Contributed equally

Key words: lung adenocarcinoma, weighted gene co-expression network analysis, risk score, least absolute shrinkage and selection operator Cox regression model and the leading cause of cancer-associated mortality worldwide (1). During the 15-year period 2000-2014, the 5-year survival rate of lung cancer was $10-20 \%$ in most countries (2). Lung adenocarcinoma (LAC), a type of non-small cell lung cancer (NSCLC), is the most diagnosed histological subtype of lung cancer (3). Diagnostic imaging is currently the main detection method for NSCLC, and the recommended treatment and the prognosis for patients with NSCLC are largely based on clinical evidence or pathological Tumor-Node-Metastasis (TNM) stage (4-6). However, the prognoses of patients with same-stage tumors may differ (7) and the underlying tumorigenic mechanism responsible for LAC remains unclear.

High-throughput technology has provided new methods for researching the molecular characterization and therapeutic targets of diseases. An improved understanding of the molecular characterization of LAC would contribute to its diagnosis, prognosis, prediction, disease monitoring and emerging therapies. The Cancer Genome Atlas (TCGA) (8) is a publicly available dataset containing genomic and clinical information on numerous types of cancer. The weighted gene co-expression network analysis (WGCNA) (9) is a free-scale network construction method suitable for dividing highly correlated genes into modules and joining these modules to external clinical traits (9), which has advantages over numerous methods in terms of global network construction (10), and has been used to assign highly co-expressed genes to several modules. The analysis has been applied in the construction of a gene network for numerous different types of cancer, such as breast (11), lung (12) and gastric (13) cancer.

In addition, the least absolute shrinkage and selection operator (LASSO) $(14,15)$ is a penalized regression method that could be used to analyze gene expression profiles. Due to its high dimensionality and high collinearity (16), the LASSO Cox regression model could be combined with the WGCNA to identify biomarkers. A previous study investigated the network-based signature of LAC in non-smokers using WGCNA and LASSO regression, and generated a 17-gene-signature that could discriminate the high-risk subgroup from the low-risk subgroup by survival analysis (12). However, differentially expressed genes (DEGs) were filtered from assigned Gene Expression Omnibus (GEO) datasets and submitted to WGCNA, which may result in compromised scale-free topology assumption. 
Therefore, in the present study, genes of LAC samples from TCGA were filtered for WGCNA according to a threshold for average gene expression value, instead of via differential expression analysis, and the LASSO Cox regression model was used to detect potential prognostic markers from the selected module thereafter.

\section{Materials and methods}

Gene expression data and clinical data. Gene expression data and clinical data for patients with LAC were obtained from TCGA (https://cancergenome.nih.gov/) on May 20, 2018 , including data from 515 LAC samples. The retrieval condition was (Program Name IS TCGA) AND (Project Id IS TCGA-LUAD) AND (Workflow Type IS HTSeq-FPKM) AND (Experimental Strategy IS RNA-Seq). Information on gene expression levels measured via RNA sequencing, denoted by fragments per kilobase of transcript per million mapped reads (FPKM), was collected. FPKM $=10^{9} \mathrm{x}$ number of reads mapped to the gene/(number of reads mapped to all protein-coding genes $\mathrm{x}$ length of the gene in base pairs). Clinical information, including pathological TNM stage and follow-up information, was also collected.

\section{WGCNA}

Network construction and module detection. A WGCN was constructed using the package WGCNA 1.63 in R (version 3.5.2). The adjacent coefficient $\left(a_{i j}\right)$ was calculated by the absolute value of Pearson's correlation coefficient of genes $i$ and $j$ to the $\beta$ th power, $a_{i j}=\left|\operatorname{cor}\left(x_{i}, x_{j}\right)\right|^{\beta}$, where $x_{i}$ is the series of expression values for gene i. $\mathrm{P}<0.05$ in the Pearson's correlation analysis was considered statistically significant. The lowest power $\beta$ was chosen when the scale-free topology fit index curve flattens out upon reaching a high value. In addition to considering the connection between two correlated genes, WGCNA also takes into account associated genes, and the topological overlaps $\left(\mathrm{T}_{\mathrm{ij}}\right)$ are calculated from $\mathrm{a}_{\mathrm{ij}}$ as follows, to compose a topological overlap matrix (TOM), as a similarity evaluation reflecting relevancy and overlap between genes:

$$
\begin{aligned}
& T_{i j}=\left\{\begin{array}{cc}
\frac{l_{i j}+a_{i j}}{\min \left\{k_{i}, k_{j}\right\}+1-a_{i j}}, \quad i \neq j \\
1 \quad, \quad i=j
\end{array}\right. \\
& l_{i j}=\sum_{u \neq i, j} a_{i u} a_{u j}, \quad k_{i}=\sum_{u \neq i} a_{i u}
\end{aligned}
$$

In these formulae, $\mathrm{u}$ represents common genes linking genes $\mathrm{i}$ and $\mathrm{j}$ together, and $\mathrm{T}_{\mathrm{ij}}$ takes into account the overlap between neighboring genes of genes $i$ and $j$. TOM was subtracted from one and converted into a topological overlap dissimilarity matrix referred to as the corresponding dissimilarity of TOM (dissTOM). A hierarchical clustering tree (dendrogram) of genes was then created based on the dissTOM. Finally, modules of highly correlated and co-expressed genes were created via a Dynamic Tree Cut algorithm (17).

Associating modules with external clinical traits and identifying hub genes. Correlations between modules and clinical traits, including pathological stage and survival time, were estimated using Spearman's correlation tests. Significantly correlated module was preserved and visualized using Cytoscape 3.6.1 (18). Genes with multiple associations were defined as hub genes.

Gene Ontology $(G O)$ and pathway-enrichment analysis. The present study investigated the potential biological functions and signaling pathways of the genes in the selected module by assessing enrichment using Gene Ontology (GO) terms and Kyoto Encyclopedia of Genes and Genomes (KEGG) pathways in Metascape (http://metascape.org) (19).

LASSO Cox regression model construction. LASSO Cox regression models were constructed using the package glmnet 2.0-16 in R. By utilizing several hub genes from the selected module, the function returns a series of values of $\lambda$ and models. The coefficients of the majority of the original genes were penalized to zero in line with the increasing values of the tuning parameter $\lambda$. The $\lambda$ was chosen when the partial likelihood deviance reached its lowest. A suitable model was chosen based on the 10-fold cross-validation of the function cv.glmnet. Using the function lambda.min, the remaining genes with non-zero LASSO coefficients were obtained. The risk score for each patient with LAC was calculated using the linear combination of each FPKM of the gene $\left(\mathrm{G}_{\mathrm{k}}\right)$ multiplied by the LASSO coefficient $\left(\mathrm{c}_{\mathrm{k}}\right)$ : Risk score $=\sum_{k=1}^{n} G_{k} \mathrm{x} c_{k}$.

Statistical analysis. Statistical analyses were conducted using SPSS software (version 20.0; IBM Corp.). Receiver operating characteristic (ROC) curves were drawn and the area under the curve (AUC) was calculated to predict 3-year survival rate. The cut-off risk score was decided when the Youden index (sensitivity + specificity-1) in the ROC curve was highest. The samples were then divided into high- and low-risk groups according to the cut-off. Survival was compared between the high- and low-risk groups using Kaplan-Meier analysis and log-rank tests. Hazard ratios (HRs) were calculated using univariate and multivariable Cox regression analysis. In a multivariate Cox regression analysis using backward selection to test the independent significance of different factor, $\mathrm{P}>0.10$ was used to remove non-significant variables from the analysis.

\section{Results}

Data preprocessing. A total of 515 samples from patients diagnosed between the ages of 33 and 88 years and classified as stage IA-IV were collected from TCGA, and 498 samples with both gene expression and clinical information were used for subsequent study. Based on the pathological TNM stage, these samples were divided into a training set and a validation set by stratified randomization, in a ratio of 7:3. A total of 127 samples from patients in the training set who completed the follow-up were subjected to sample clustering, and 4 outlier samples were removed prior to the network construction (Fig. 1A). The threshold for average gene expression value was set as 1. Protein-coding genes with average expression values less than the threshold value in all samples were excluded. The final training set was comprised of 348 samples and the validation set was comprised of 150 samples. Data for a total of 123 samples, including the expression levels of 12,914 

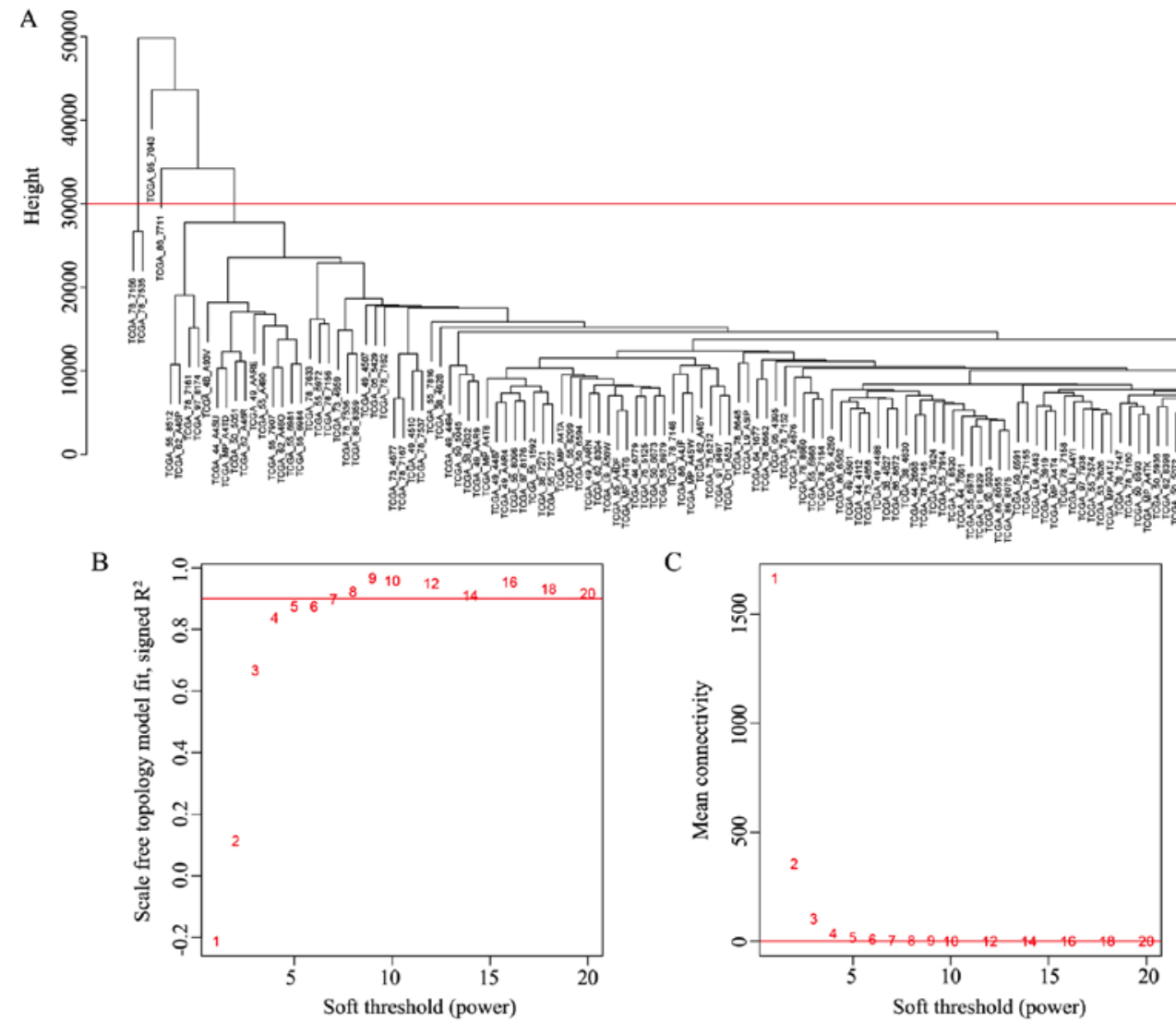

Figure 1. Sample clustering and $\beta$ decision. (A) Clustering dendrogram. Clustering dendrogram of samples based on their Euclidean distance. There were four outlier samples that were removed. (B) Scale-free topology fit $\mathrm{R}^{2}$ and series of soft thresholds. The red line indicates an $\mathrm{R}^{2}$ value of 0.90 . (C) Mean connectivity and series of soft thresholds. The red line indicates a mean connectivity value of 0 .

protein-coding genes and clinical information, were obtained for the WGCNA.

WGCN of LAC. When the soft thresholding power $\beta$ was set as 7 , the scale-free topology fit index curve flattened out at 0.90 (Fig. 1B and C). The constructed weighted gene co-expression network included 42 modules, including 39-1,360 genes. The grey module included genes that did not belong to any other modules (Fig. 2A).

Identifying modules with clinical significance. The present study analyzed the correlations between each module and clinical traits, including pathological TNM stage and survival information. In the modules, the pale turquoise module was positively and the light yellow, light green, royal blue, red, light cyan modules were negatively correlated with pathological TNM stage, whereas the light green module was positively and the violet, dark red, pink, yellow green, white, orange red modules were negatively correlated with survival time. $\mathrm{P}<0.05$ in the Spearman's correlation tests was considered statistically significant (Fig. 2Ba and b).

The dark red module was further analyzed, and it was revealed to have the strongest negative correlation with survival time (Spearman's correlation-0.33; $\mathrm{P}<0.01$ ), but was not considered significantly correlated with pathological TNM stage (Spearman's correlation $0.18 ; \mathrm{P}=0.05$ ). Therefore, it was possible to detect prognostic factors independent of staging in the dark red module. A total of 20 genes with $>20$ associations were defined as hub genes in the dark red module, including C5AR2, MUCL1, MME, KRT16, S100A12, ACKR3, SYDE1, CDK5RAP2, DIO2, SOWAHC, COA6, PTGR1, OPN3, GALNT2, FAM83A, RSPO3, ZC3H12A, KRT6A, CD36 and KYNU. The gene network of the dark red module was visualized using Cytoscape (Fig. 3).

Functional characterization of genes in the dark red module. In order to investigate the functional significance of the identified genes in the dark red module, 113 genes were subjected to GO term and KEGG pathway enrichment analyses. Among the genes most negatively correlated with survival time, 'multicellular organismal homeostasis' ( $\log P,-15.2)$, 'adenylate cyclase activating pathway' ( $\log \mathrm{P},-15.1)$ and 'fatty acid metabolism' $(\log \mathrm{P},-14.8)$ were also the most significantly enriched genes in the GO term and KEGG pathway enrichment analyses (Fig. 4).

Prognostic signature construction via LASSO Cox regression model using the training set. The LASSO Cox regression model was constructed using the glmnet package in $\mathrm{R}$ by utilizing several hub genes in the dark red module. Based on the 10 -fold cross-validation, the value 0.087 was chosen as the minimum criteria for $\lambda$. At the $\lambda$ parameter, the total absolute of non-zero coefficients was 0.0214 and there were four genes (OPN3, GALNT2, FAM83A and KYNU) obtained with non-zero coefficients (Fig. 5A and B). Based on the genes with non-zero coefficients, the risk score of every patient was calculated according to the linear combination of each gene 


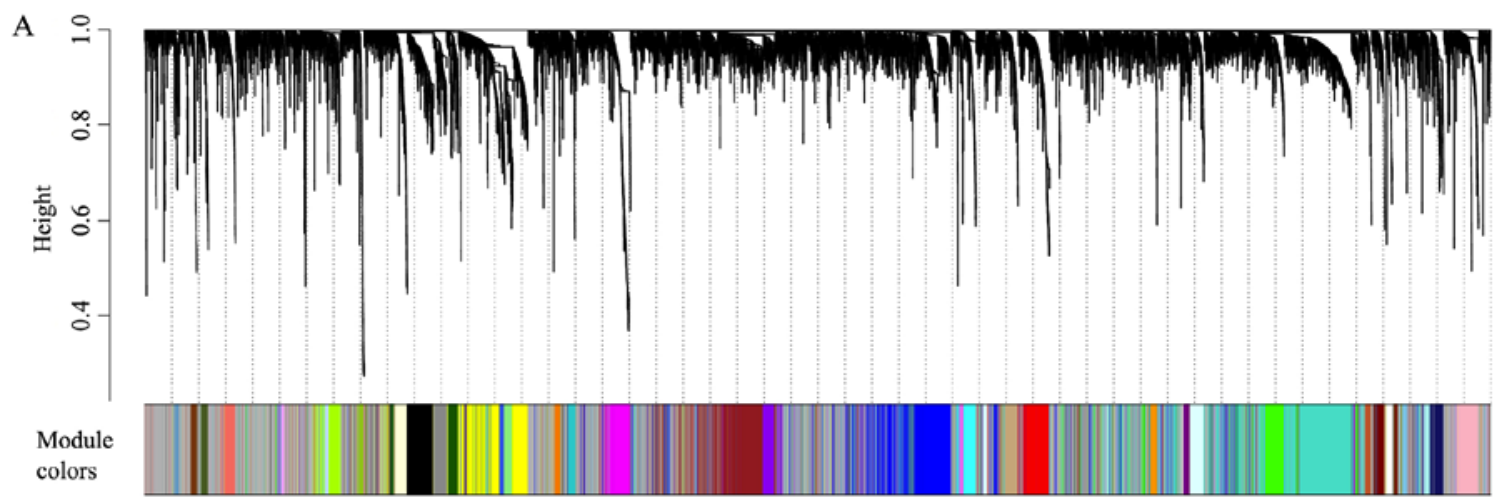

B
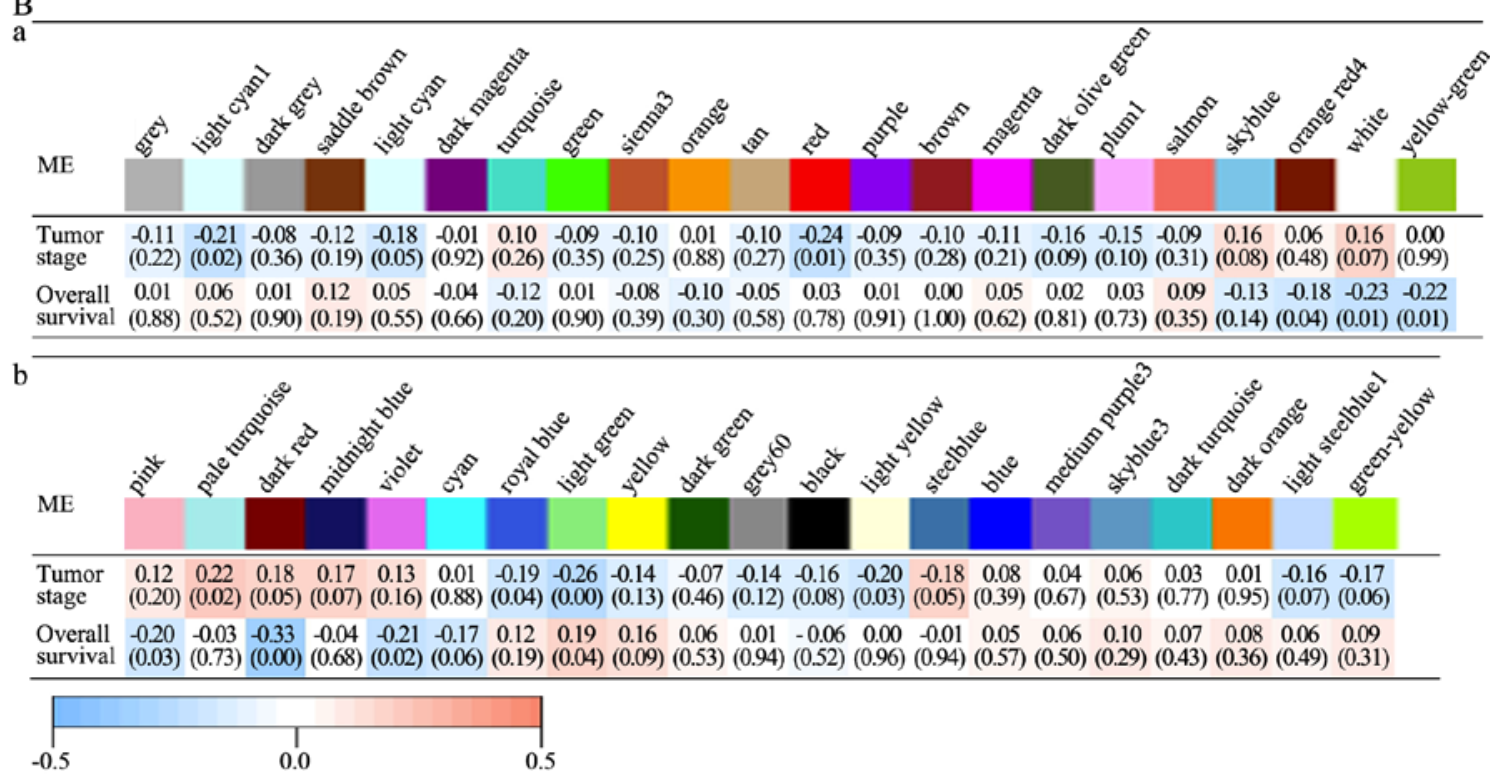

Figure 2. All 42 modules and their module-trait associations identified by the WGCNA. (A) The WGCN of LAC identified 42 modules with correlated genes. A dendrogram was produced based on the WGCNA package in R by average linkage hierarchical clustering of 12,914 protein-coding genes. $(\mathrm{Ba}$ and $\mathrm{b})$ Module-trait associations. Each column represents a module eigengene and each row represents a clinical trait. Each cell contains the correlation coefficient (first line) and P-value (in parentheses). The figures are drawn according to the color legend: Pale turquoise module was positively correlated with pathological TNM stage; light yellow, light green, royal blue, red and light cyan modules were negatively correlated with pathological TNM stage; light green module was positively correlated with survival time; and violet, dark red, pink, yellow green, white, and orange red modules were negatively correlated with survival time. $\mathrm{P}<0.05$ in the Spearman's correlation tests was considered to indicate a statistically significant result. WGCNA, weighted gene co-expression network analysis; LAC, lung adenocarcinoma; TNM, Tumor-Node-Metastasis; ME, module eigengene.

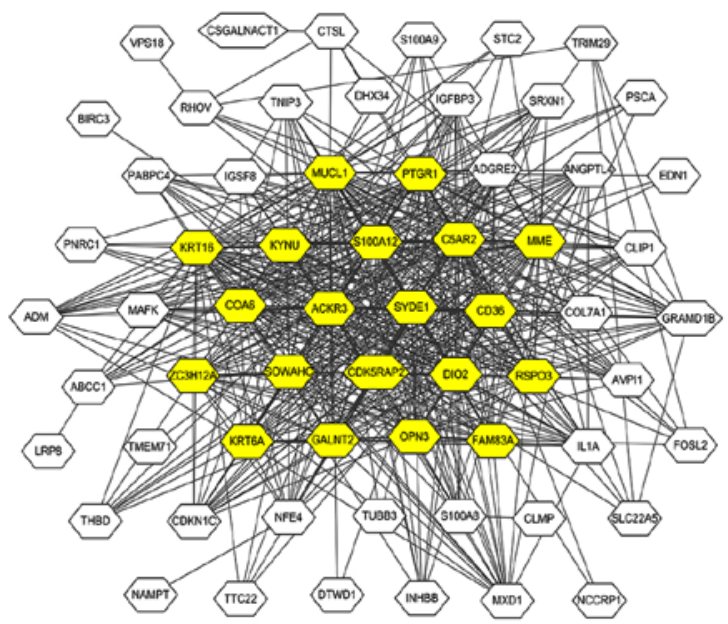

Figure 3. Co-expression network of the dark red module. There were 63 connected genes in the dark red module. Nodes are genes and lines represent their connections. The 20 yellow nodes are the hub genes of the network. There were 50 genes not presented in the network, as their connections with other genes were not strong enough to reach the threshold. expression multiplied by the LASSO coefficients: $(0.0004 \mathrm{x}$ OPN3 $)+(0.0042 \times$ GALNT2 $)+(0.0055 \times$ FAM83A $)+(0.0077$ $\mathrm{x}$ KYNU).

Survival analysis. ROC curves were used to assess the prognostic power based on OS at 3 years (Fig. 6A). The cut-off risk score was determined to be 0.216 . Patients in the high-risk group had significantly poorer OS time than those in the low-risk group. The mean OS time was 44.6 months [95\% confidence interval (CI), 35.9-53.3] in the high-risk group and 95.0 months (95\% CI, 73.2-116.9) in the low-risk group $(\mathrm{P}<0.001$; Fig. 6B).

The results were similar for the validation set. The mean OS time was 48.4 months (95\% CI, 35.0-61.7) in the high-risk group and 93.2 months $(95 \% \mathrm{CI}, 71.7-114.6)$ in the low-risk group $(\mathrm{P}=0.016$; Fig. 6C).

Risk was then verified as an independent prognostic factor for OS. Univariable and multivariable analyses of potential prognostic factors in the total set for OS were performed. The risk score and TNM stage were associated with OS in 


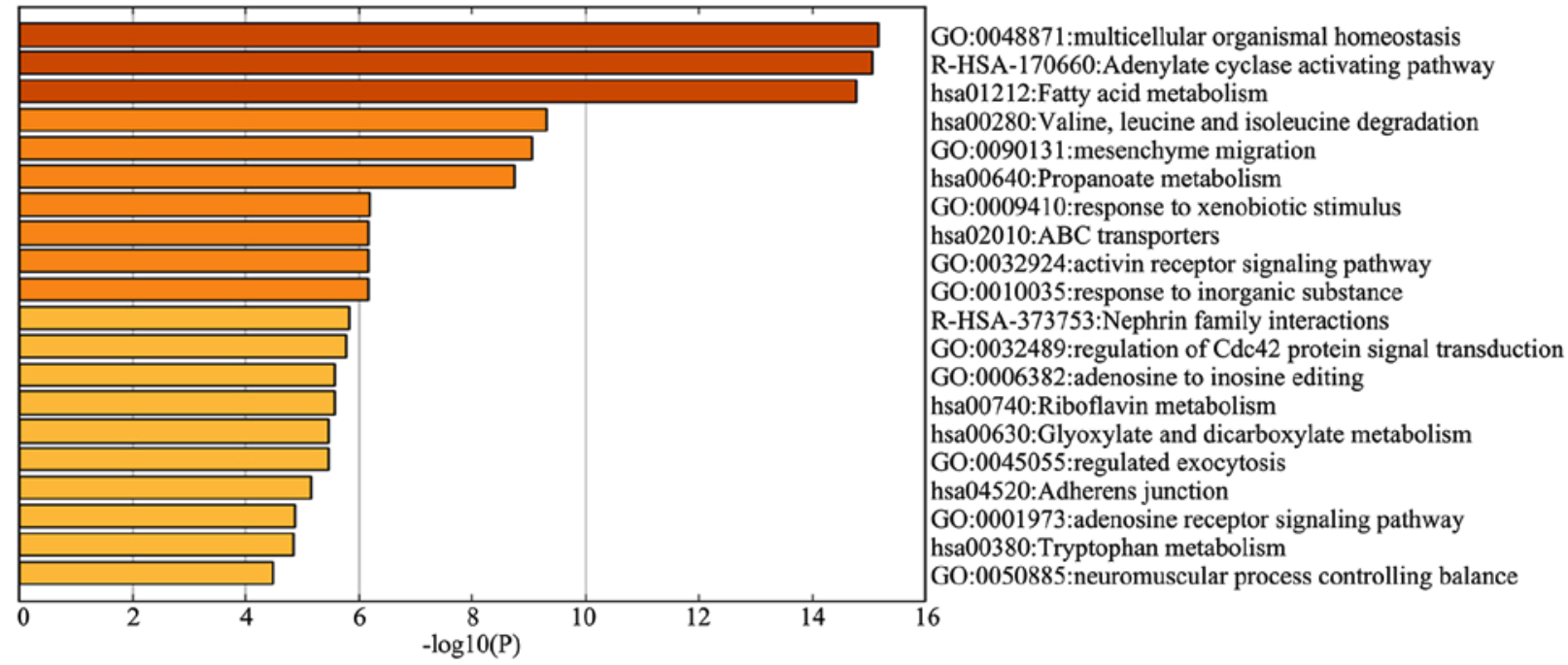

Figure 4. Top 20 clusters in the dark red module. Heatmap of top 20 clusters, colored according to P-value.

A

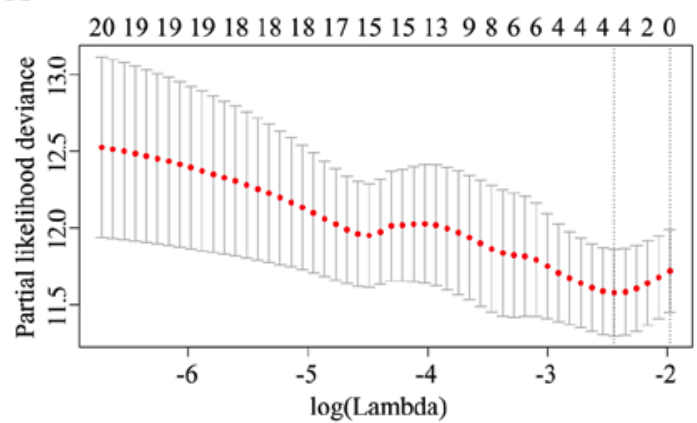

B

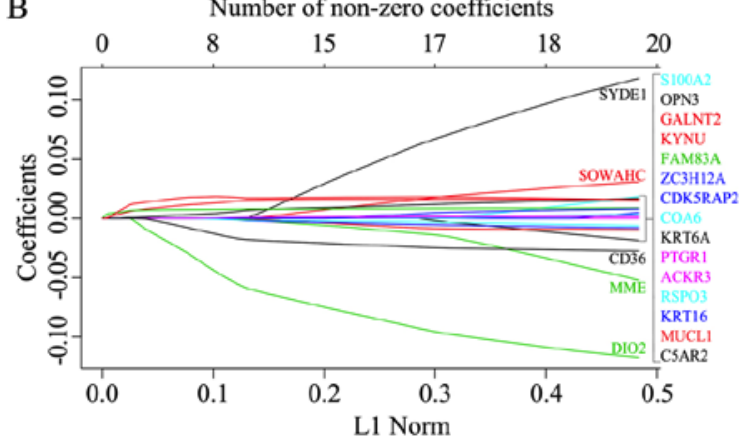

Figure 5. LASSO Cox regression model construction. (A) $\lambda$ selection by 10 -fold cross-validation. Continuous upright lines are partial likelihood deviance $\pm \mathrm{SE}$; dotted lines are depicted at the optimal values by minimum criteria (lambda.min, left vertical dotted line) and 1-SE criteria (lambda.1se, right vertical dotted line). The partial likelihood deviance with changing of $\log (\lambda)$ was plotted. The value 0.087 was chosen for $\lambda$ by 10 -fold cross-validation with the minimum criteria. (B) Processes of LASSO Cox model fitting. Each curve represents a gene. The trend of each coefficient against the L1-norm is plotted when $\lambda$ changes. L1-norm is the total absolute of non-zero coefficients. LASSO, least absolute shrinkage and selection operator; SE, standard error.

the univariable analysis. Following the multivariable analysis, the risk score and TNM stage remained prognostic factors for OS (Table I). Patients with LAC who had a high-risk score experienced poorer OS time (HR, 1.699; 95\% CI, 1.242-2.324; $\mathrm{P}=0.001)$.
A

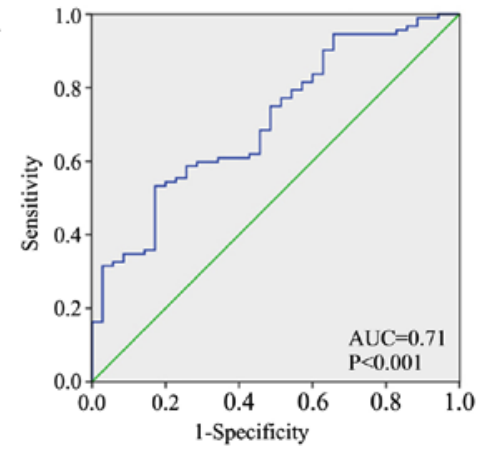

B

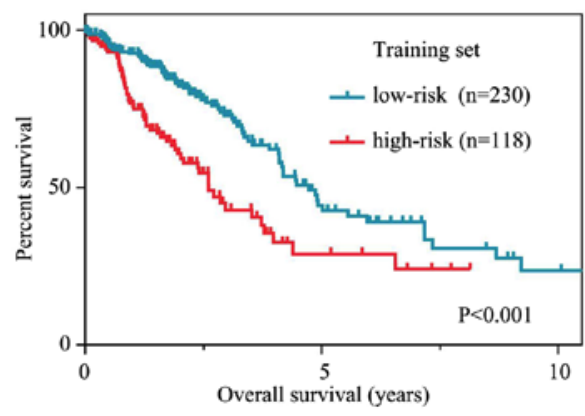

C

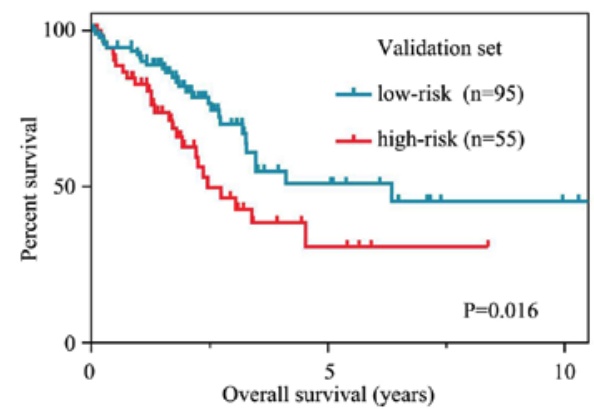

Figure 6. Cut-off risk score decision and LASSO Cox regression model validation. (A) The receiver operator characteristic was drawn and the AUC was calculated. The AUC was 0.71 (95\% CI, 0.61-0.81) to predict 3-year survival using the training set. The cut-off risk score was determined to be 0.216. (B) Survival comparison between the high- and low-risk groups of the training set using Kaplan-Meier analysis and log-rank tests. (C) Survival comparison between the high- and low-risk groups of the validation set using Kaplan-Meier analysis and log-rank tests. LASSO, least absolute shrinkage and selection operator; AUC, area under the curve. 
Table I. Univariable and multivariable Cox regression analysis of prognosis factors in the total set for OS.

\begin{tabular}{|c|c|c|c|c|c|c|}
\hline \multirow[b]{2}{*}{ OS variable } & \multicolumn{3}{|c|}{ Univariate analysis } & \multicolumn{3}{|c|}{ Multivariable analysis } \\
\hline & HR & $95 \% \mathrm{CI}$ & P-value & HR & $95 \% \mathrm{CI}$ & P-value \\
\hline Risk score (high vs. low) & 1.989 & $1.478-2.679$ & $<0.001$ & 1.699 & $1.242-2.324$ & 0.001 \\
\hline \multicolumn{7}{|l|}{ TNM stage } \\
\hline (Stage II vs. I) & 2.468 & $1.710-3.562$ & $<0.001$ & 2.111 & $1.437-3.103$ & $<0.001$ \\
\hline (Stage III vs. I) & 3.623 & $2.473-5.307$ & $<0.001$ & 2.973 & 2.009-4.399 & $<0.001$ \\
\hline (Stage IV vs. I) & 3.880 & $2.234-6.740$ & $<0.001$ & 3.355 & $1.929-5.845$ & $<0.001$ \\
\hline Age ( $\geq 60$ vs. $<60$ years) & 1.007 & $0.723-1403$ & 0.967 & & & \\
\hline
\end{tabular}

HR, hazard ratio; CI, confidence interval; OS, overall survival; TNM, Tumor-Node-Metastasis.

Clinical and pathological TNM staging serves an important role in predicting the prognosis of patients with LAC. The 5-year survival rate is $77-92 \%$ for clinical stage IA, $68 \%$ for stage IB; $60 \%$ for stage IIA and $53 \%$ for stage IIB. For pathological stage, the 5-year survival is $80-90 \%$ for stage IA, $73 \%$ for stage IB, $65 \%$ for stage IIA and $56 \%$ for stage IIB (6). In the present study, when comparing TNM stage on multivariable analysis, stage II (HR, 2.111), stage III (HR, 2.973) and stage IV (HR, 3.355) patients had poorer OS compared with stage I patients (all $\mathrm{P}<0.001$ ). Age had no effect on LAC prognosis (Table I).

\section{Discussion}

In the present study, a survival-associated risk score for LAC was identified using the combination of WGCNA and the LASSO Cox regression model in order to investigate a new molecular characterization of LAC that was associated with prognosis, and a risk model was produced to aid its diagnosis and management. A training set consisting of 348 LAC samples with gene expression and clinical information was analyzed to detect survival-associated biomarkers, and the results were verified in a validation set comprising $150 \mathrm{LAC}$ samples. The results from the present study suggested that these four novel markers could effectively be of diagnostic and therapeutic value for the management of LAC.

WGCNA provides a comprehensive set of functions for performing a weighted correlation network analysis, and is designed to construct a gene network at very large scales (9). The Cox regression model (20) is used for regression analyses of censored survival data. However, the standard maximum Cox partial likelihood method cannot be applied directly to genes with very high dimensionality and highly correlated expression levels (16). Tibshirani (14) expanded the LASSO method for variable selection in the Cox model and proposed minimizing the log partial likelihood subject to the sum of the absolute values of the parameters being bounded by a constant in order to obtain the parameter estimates. This method facilitates model fitting in situations where there are as many, or even more, explanatory variables than there are observations, and only a few variables are relevant to explaining the data (21). As long as the training set is not smaller than the number of predictors, this procedure can be applied directly to the genes selected by WGCNA.

Recent research constructed prognostic models for LAC based on the gene expression data, with the aim of improving its early diagnosis and personalized treatment (22). Zhao et al (23) integrated differential expression and regression analyses for LAC datasets from TCGA and GEO. The AUCs of their 4-gene and 20-gene models were 0.5731 and 0.615 , respectively. Mao et al (12) considered DEGs in non-smokers with LAC and defined the threshold for DEGs as a fold-change $>2$, with an adjusted P-value of $<0.05$. DEGs from the assigned datasets were submitted to WGCNA. This study highlighted two gene modules associated with non-smoking LAC through WGCNA and built a prognostic signature with 17 candidate genes, which provided a novel compendium of biomarkers to act as a guide for therapy in non-smokers with LAC (12). However, these previous models did not consider protein-coding genes that were below the threshold for DEGs. Being designed to be an unsupervised analysis method, WGCNA clusters genes based on their expression profiles. Since low-expression or non-varying genes usually represent noise, it is suggested to filter genes by mean expression or variance. Filtering genes by differential expression is not recommended, as it would invalidate the scale-free topology assumption leading to the creation of a few highly correlated modules. In addition, it fails to select soft thresholding power by scale-free topology (24).

Studies regarding the use of liquid biopsies, such as tumor-educated platelets, cell-free DNA, circulating tumor cells and extracellular vesicles, have markedly increased in number and may radically change the future management of tumors $(22,25,26)$. In the present study, the LASSO Cox regression model construction of the hub genes in the dark red module identified OPN3, GALNT2, FAM83A and KYNU as the most valuable genes associated with LAC survival. The risk score tends to be larger with increasing expression of these genes, and patients with LAC that had high risk scores had significantly poorer OS times. However, to the best of our knowledge, there are relatively few studies regarding these genes, particularly OPN3. It cannot yet be concluded that they play an important role in the survival of patients with LAC. OPN3 is highly expressed in the brain and testes, and weakly expressed in the liver, placenta, heart, lung, skeletal muscle, kidney and pancreas (27). Acquired resistance to 5-fluorouracil 
in hepatocellular carcinoma cells can be reversed by overexpression of OPN3 (28). Yoshimoto et al (29) identified that OPN3-knockdown reversed the effect of decreased colon cancer cell viability following blue LED irradiation. GALNT2 encodes the polypeptide $\mathrm{N}$-acetylgalactosaminyltransferase 2 , which is involved in O-linked protein glycosylation (30). GALNT2 has been identified as a candidate gene in lipid metabolism by genome-wide association studies, and its single nucleotide polymorphisms may be correlated with plasma lipids $(31,32)$. The overexpression of GALNT2 can promote the invasive potential of oral squamous cell carcinoma (OSCC) cells by modifying the O-glycosylation of proteins and increasing the activity of epidermal growth factor receptor (EGFR), which plays an important role in the invasive behavior of OSCC cells (33). Imielinski et al (34) confirmed the high mutation rate of EGFR $(17 \%)$ in LAC. Therefore, whether the role of GALNT2 in the glycosylation of O-protein is involved in the occurrence and development of LAC disease remains to be further studied. FAM83A encodes the protein family member with sequence similarity 83 (FAM83A), also known as tumor antigen BJ-TSA-9. Lee et al (35) identified that FAM83A was a candidate cancer-associated gene capable of conferring resistance to EGFR-tyrosine kinase inhibitors, and that FAM83A interacted with and caused phosphorylation of c-RAF and phosphoinositide 3 kinase p85, upstream of MAPK and downstream of EGFR in breast cancer cells and in mice (35). Li et al (36) identified a tumor-specific antigen, TSA-9, which was highly expressed in lung cancer tissues. Liu et al (37) detected circulating cancer cells in lung cancer patients using a panel of marker genes including BJ-TSA-9. In addition, cigarette smoking can induce the expression of FAM83A (38). KYNU encodes kynureninase, which is involved in the biosynthesis of nicotinamide adenine dinucleotide cofactors from tryptophan. KYNU expression was demonstrated to be decreased in invasive ductal carcinoma and osteosarcoma cell lines compared with normal fibroadenoma $(39,40)$. KYNU may be associated with metabolic transformation in cancer development. The transition from oxidative phosphorylation to aerobic glycolysis is a sign of stem cell function in normal tissue growth and differentiation. Overall, further in vivo and in vitro experiments are required in order to clarify the roles of OPN3, GALNT2 and KYNU in lung cancer, and the clinical significance of FAM83A in LAC requires further investigation.

However, the prognostic model of the present study had certain limitations. First, further validation, such as a reverse transcription-quantitative PCR validation, in an independent set is required in order to confirm the diagnostic value of the model used. Secondly, other clinical information that may have influenced OS was not obtained, such as primary health problems and follow-up treatment. Thirdly, it is uncertain as to whether the risk score is feasible for use with metastatic tumors, as the samples used in the present study were from primary tumors, the initial site of cancer. The risk score was obtained from the dark red module, which demonstrated the strongest negative correlation with survival time. Further investigations are required in order to detect the markers from other modules.

In conclusion, WGCNA and LASSO Cox regression analysis were applied to the LAC data from TCGA, and a four-gene-based risk score was obtained that may support the development of diagnostic and therapeutic strategies for LAC management.

\section{Acknowledgements}

Not applicable.

\section{Funding}

The present study was supported by the President Foundation of Nanfang Hospital, Southern Medical University, Guangzhou, China (grant no. 2016B018).

\section{Availability of data and materials}

The datasets used and/or analyzed during the present study are available from the corresponding author upon reasonable request, and from TCGA (https://cancergenome.nih.gov/).

\section{Authors' contributions}

$\mathrm{KC}$ was a major contributor in designing the study. HeW, DL and XGL performed the data analyses. HeW was a major contributor in writing the manuscript. JJJ suggested analysis of the data based on stratified randomization and was a contributor in writing the manuscript. SYF contributed to data collection and data analysis. XYD and XSS were responsible for data pretreatment. HFW contributed to the conception of the study, and was responsible for figure processing, table generation and discussion drafting. HuW and GX revised the manuscript and interpreted the data. All authors read and approved the final manuscript.

\section{Ethics approval and consent to participate}

Not applicable.

\section{Patient consent for publication}

Not applicable.

\section{Competing interests}

The authors declare that they have no competing interests.

\section{References}

1. Torre LA, Bray F, Siegel RL, Ferlay J, Lortet-Tieulent J and Jemal A: Global cancer statistics, 2012. CA Cancer J Clin 65: 87-108, 2015.

2. Allemani C, Matsuda T, Di Carlo V, Harewood R Matz M, Nikšić M, Bonaventure A, Valkov M, Johnson CJ, Estève J, et al: Global surveillance of trends in cancer survival 2000-14 (CONCORD-3): Analysis of individual records for 37513025 patients diagnosed with one of 18 cancers from 322 population-based registries in 71 countries. Lancet 391: 1023-1075, 2018.

3. Sanchez-Salcedo P, Berto J, de-Torres JP, Campo A, Alcaide AB, Bastarrika G, Pueyo JC, Villanueva A, Echeveste JI, Lozano MD, et al: Lung cancer screening: Fourteen year experience of the Pamplona early detection program (P-IELCAP). Arch Bronconeumol 51: 169-176, 2015 (In English, Spanish). 
4. Vansteenkiste J, Crinò L, Dooms C, Douillard JY, Faivre-Finn C, Lim E, Rocco G, Senan S, Van Schil P, Veronesi G, et al: 2nd ESMO Consensus conference on lung cancer: Early-stage non-small-cell lung cancer consensus on diagnosis, treatment and follow-up. Ann Oncol 25: 1462-1474, 2014.

5. Hirsch FR, Scagliotti GV, Mulshine JL, Kwon R, Curran WJ Jr, Wu YL and Paz-Ares L: Lung cancer: Current therapies and new targeted treatments. Lancet 389: 299-311, 2017.

6. Goldstraw P, Chansky K, Crowley J, Rami-Porta R, Asamura H, Eberhardt WE, Nicholson AG, Groome P, Mitchell A, Bolejack V, et al: The IASLC lung cancer staging project: Proposals for revision of the TNM stage groupings in the forthcoming (Eighth) edition of the TNM classification for lung cancer. J Thorac Oncol 11: 39-51, 2016.

7. Chansky K, Sculier JP, Crowley JJ, Giroux D, Van Meerbeeck J and Goldstraw P; International Staging Committee and Participating Institutions: The international association for the study of lung cancer staging project: Prognostic factors and pathologic TNM stage in surgically managed non-small cell lung cancer. J Thorac Oncol 4: 792-801, 2009.

8. Cancer Genome Atlas Research Network; Weinstein JN, Collisson EA, Mills GB, Shaw KR, Ozenberger BA, Ellrott K, Shmulevich I, Sander C and Stuart JM: The cancer genome atlas pan-cancer analysis project. Nat Genet 45: 1113-1120, 2013

9. Langfelder $P$ and Horvath S: WGCNA: An R package for weighted correlation network analysis. BMC Bioinformatics 9 : $559,2008$.

10. Allen JD, Xie Y, Chen M, Girard L and Xiao G: Comparing statistical methods for constructing large scale gene networks PLoS One 7: e29348, 2012.

11. Guo L, Zhang K and Bing Z: Application of a co-expression network for the analysis of aggressive and non-aggressive breas cancer cell lines to predict the clinical outcome of patients. Mol Med Rep 16: 7967-7978, 2017.

12. Mao Q, Zhang L, Zhang Y, Dong G, Yang Y, Xia W, Chen B, $\mathrm{Ma} \mathrm{W}, \mathrm{Hu} J$, Jiang F and $\mathrm{Xu} \mathrm{L}$ : A network-based signature to predict the survival of non-smoking lung adenocarcinoma. Cancer Manag Res 10: 2683-2693, 2018.

13. Chen J, Wang X, Hu B, He Y, Qian X and Wang W: Candidate genes in gastric cancer identified by constructing a weighted gene co-expression network. PeerJ 6: e4692, 2018.

14. Tibshirani R: The lasso method for variable selection in the Cox model. Stat Med 16: 385-395, 1997.

15. Tibshirani R: Regression shrinkage and selection via the lasso. J R Stat Soc Ser A Stat Soc 73: 273-282, 2011.

16. Gui J and Li H: Penalized Cox regression analysis in the high-dimensional and low-sample size settings, with applications to microarray gene expression data. Bioinformatics 21 : 3001-3008, 2005.

17. Langfelder P, Zhang B and Horvath S: Defining clusters from a hierarchical cluster tree: The dynamic tree cut package for $\mathrm{R}$ Bioinformatics 24: 719-720, 2008.

18. Shannon P, Markiel A, Ozier O, Baliga NS, Wang JT, Ramage D, Amin N, Schwikowski B and Ideker T: Cytoscape: A software environment for integrated models of biomolecular interaction networks. Genome Res 13: 2498-2504, 2003.

19. Tripathi S, Pohl MO, Zhou Y, Rodriguez-Frandsen A, Wang G, Stein DA, Moulton HM, DeJesus P, Che J, Mulder LC, et al: Meta- and orthogonal integration of influenza 'OMICs' data defines a role for UBR4 in virus budding. Cell Host Microbe 18 723-735, 2015.

20. Cox DR: Regression models and life-tables. Springer New York, 1992.

21. Pasanen L, Holmström L and Sillanpää MJ: Bayesian LASSO, scale space and decision making in association genetics. PLoS One 10: e0120017, 2015.

22. Bracht JWP, Mayo-de-Las-Casas C, Berenguer J, Karachaliou N and Rosell R: The present and future of liquid biopsies in non-small cell lung cancer: Combining four biosources for diagnosis, prognosis, prediction, and disease monitoring. Curr Oncol Rep 20: 70, 2018.

23. Zhao K, Li Z and Tian H: Twenty-gene-based prognostic model predicts lung adenocarcinoma survival. OncoTargets Ther 11: 3415-3424, 2018.
24. Langfelder $P$ and Horvath S: WGCNA package FAQ https://horvath.genetics.ucla.edu/html/CoexpressionNetwork/ Rpackages/WGCNA/faq.html, 2017. Accessed December 24, 2017

25. Friedrich MJ: Going with the flow: The promise and challenge of liquid biopsies. JAMA 318: 1095-1097, 2017.

26. Siravegna G, Marsoni S, Siena S and Bardelli A: Integrating liquid biopsies into the management of cancer. Nat Rev Clin Oncol 14: 531-548, 2017.

27. Fagerberg L, Hallström BM, Oksvold P, Kampf C, Djureinovic D, Odeberg J, Habuka M, Tahmasebpoor S, Danielsson A, Edlund $\mathrm{K}$, et al: Analysis of the human tissue-specific expression by genome-wide integration of transcriptomics and antibody-based proteomics. Mol Cell Proteomics 13: 397-406, 2014.

28. Jiao J, Hong S, Zhang J, Ma L, Sun Y, Zhang D, Shen B and Zhu C: Opsin3 sensitizes hepatocellular carcinoma cells to 5 -fluorouracil treatment by regulating the apoptotic pathway. Cancer Lett 320: 96-103, 2012.

29. Yoshimoto T, Morine Y, Takasu C, Feng R, Ikemoto T, Yoshikawa K, Iwahashi S, Saito Y, Kashihara H, Akutagawa M, et al: Blue light-emitting diodes induce autophagy in colon cancer cells by Opsin 3. Ann Gastroenterol Surg 2: 154-161, 2018.

30. Yang B, Yan S, Yan J, Li Y, Khurwolah MR, Wang L and Chen Z: A study of the association of rs12040273 with susceptibility and severity of coronary artery disease in a Chinese Han population. BMC Cardiovasc Disord 18: 10, 2018.

31. Willer CJ, Sanna S, Jackson AU, Scuteri A, Bonnycastle LL, Clarke R, Heath SC, Timpson NJ, Najjar SS, Stringham HM, et al: Newly identified loci that influence lipid concentrations and risk of coronary artery disease. Nat Genet 40: 161-169, 2008.

32. Kathiresan S, Melander O, Guiducci C, Surti A, Burtt NP, Rieder MJ, Cooper GM, Roos C, Voight BF, Havulinna AS, et al: Six new loci associated with blood low-density lipoprotein cholesterol, high-density lipoprotein cholesterol or triglycerides in humans. Nat Genet 40: 189-197, 2008.

33. Lin MC, Huang MJ, Liu CH, Yang TL and Huang MC: GALNT2 enhances migration and invasion of oral squamous cell carcinoma by regulating EGFR glycosylation and activity. Oral Oncol 50: 478-484, 2014

34. Imielinski M, Berger AH, Hammerman PS, Hernandez B, Pugh TJ, Hodis E, Cho J, Suh J, Capelletti M, Sivachenko A, et al: Mapping the hallmarks of lung adenocarcinoma with massively parallel sequencing. Cell 150: 1107-1120, 2012

35. Lee SY, Meier R, Furuta S, Lenburg ME, Kenny PA, Xu R and Bissell MJ: FAM83A confers EGFR-TKI resistance in breast cancer cells and in mice. J Clin Invest 122: 3211-3220, 2012.

36. Li Y, Dong X, Yin Y, Su Y, Xu Q, Zhang Y, Pang X, Zhang Y and Chen W: BJ-TSA-9, a novel human tumor-specific gene, has potential as a biomarker of lung cancer. Neoplasia 7: 1073-1080, 2005.

37. Liu L, Liao GQ, He P, Zhu H, Liu PH, Qu YM, Song XM, Xu QW, Gao Q, Zhang Y, et al: Detection of circulating cancer cells in lung cancer patients with a panel of marker genes. Biochem Biophys Res Commun 372: 756-760, 2008.

38. Li Y, Xiao X, Ji X, Liu B and Amos CI: RNA-seq analysis of lung adenocarcinomas reveals different gene expression profiles between smoking and nonsmoking patients. Tumour Biol 36: 8993-9003, 2015

39. Perou CM, Sørlie T, Eisen MB, van de Rijn M, Jeffrey SS, Rees CA, Pollack JR, Ross DT, Johnsen H, Akslen LA, et al: Molecular portraits of human breast tumours. Nature 406: 747-752, 2000.

40. Lauvrak SU, Munthe E, Kresse SH, Stratford EW, Namløs HM, Meza-Zepeda LA and Myklebost O: Functional characterisation of osteosarcoma cell lines and identification of mRNAs and miRNAs associated with aggressive cancer phenotypes. $\mathrm{Br}$ J Cancer 109: 2228-2236, 2013

This work is licensed under a Creative Commons

Attribution-NonCommercial-NoDerivatives 4.0 International (CC BY-NC-ND 4.0) License. 